\title{
Distributions of the values of some arithmetical functions
}

by

P. ERdös (Budapest) and A. Schinzel (Warszawa)

§ 1. Y. Wang and A. Schinzel proved, by Brun's method, the following theorem $([3])$ :

For any given sequence of $h$ non-negative numbers $a_{1}, a_{2}, \ldots, a_{h}$ and $\varepsilon>0$, there exist positive constants $c=c(a, \varepsilon)$ and $x_{0}=x_{0}(a, \varepsilon)$ such that the number of positive integers $n \leqslant x$ satisfying

$$
\left|\frac{\varphi(n+i)}{\varphi(n+i-1)}-a_{i}\right|<\varepsilon \quad(1 \leqslant i \leqslant h)
$$

is greater than $c x / \log ^{h+1} x$, whenever $x>x_{0}$.

They also proved the analogous theorem for the function $\sigma$.

Shao Pin Tsung, also using Brun's method, extended this result to all multiplicative positive functions $f_{s}(n)$ satisfying the following conditions ([4]):

I. For any positive integer $l$ and prime number $p$ :

$$
\left.\lim _{p \rightarrow \infty}\left(f_{s}\left(p^{l}\right) / p^{l s}\right)=1 \quad \text { ( } p \text { denotes primes }\right) .
$$

II. There exists an interval $\langle a, b\rangle, a=0$ or $b=\infty$, such that for any integer $M>0$ the set of numbers $f_{s}(N) / N^{s}$, where $(N, M)=1$, is dense in $\langle a, b\rangle$.

(This formulation is not the same but equivalent to the original one.)

In this paper we shall show without using Brun's method that if we replace the condition I by the condition

$$
\sum \frac{\left(f_{s}(p)-p^{s}\right)^{2}}{p^{2 s+1}}<\infty
$$

(but preserving condition II) then there exist more than $C(a, \varepsilon) x$ posi- 
tive integers $n \leqslant x$ for which

$$
\left|\frac{f_{s}(n+i)}{f_{s}(n+i-1)}-a_{i}\right|<\varepsilon \quad(i=1,2, \ldots, h) .
$$

This theorem follows easily from the following stronger theorem.

THEOREM 1. Let $f(n)$ be an additive function, satisfying the following conditions

1. $\sum_{p}\left(\|f(p)\|^{2} / p\right)$ is convergent, where $\|f\|$ denotes $f(p)$ for $|f(p)| \leqslant 1$ and 1 for $|f(p)|>1$.

2. There exists a number $c_{1}$ such that, for any integer $M>0$, the set of numbers $f(N)$, where $(N, M)=1$ is dense in $\left(c_{1}, \infty\right)$.

Then, for any given sequence of $h$ real numbers $a_{1}, a_{2}, \ldots, a_{h}$ and $\varepsilon>0$, there exist more than $C(a, \varepsilon) x$ positive integers $n \leqslant x$ for which

$$
\left|f(n+i)-f(n+i-1)-a_{i}\right|<\varepsilon \quad(i=1,2, \ldots, h) ;
$$

$C(a, \varepsilon)$ is a positive constant, depending on $\varepsilon$ and $a_{i}$.

LEMrMa. There exists an absolute constant 0 such that the number of the integers of the form $p q>x$ for which one can find $n \leqslant x$ satisfying $n \equiv b(\bmod a), n \equiv 0(\bmod p)$ and $n+1 \equiv 0(\bmod q)$ is for $x>x_{0}(a)$ less than $c x / a$.

Proof. Let $c_{1}, c_{2}, \ldots$ denote absolute constants. Assume $p>x^{1 / 2}$ $\left(q>x^{1 / 2}\right.$ can be dealt similarly). Denote by $A_{l}(x)$ the number of integers of the form $p q$ satisfying

$$
p q>x, \quad x^{1-1 / 2^{l}} \leqslant p<x^{1-1 / 2^{l+1}}, \quad n \equiv b(\bmod a), \quad p|n, q| n+1,
$$

for some $n, 1 \leqslant n \leqslant y$,

and by $A_{l}^{\prime}(x)$ the number of integers $p q$ for which

$$
\begin{aligned}
& x^{1-1 / 2^{l}} \leqslant p<x^{1-1 / 2^{l+1}}, \quad q>x^{1 / 2^{2+1}}, \quad n \equiv b(\bmod a), \quad p|n, \quad q| n+1, \\
& \text { for some } n, \quad 1 \leqslant n \leqslant x \text {. }
\end{aligned}
$$

Clearly $A_{l}^{\prime}(x) \geqslant A_{l}(x)$ and it will suffice to prove that for $x>x_{0}(a)$, $\sum_{i=1}^{\infty} A_{l}^{\prime}(x)<c x / a$.

Define positive integer $l_{x}$ by the inequality

$$
2^{l} x \geqslant \frac{1}{a} \log x>2^{l_{x-1}}
$$

The number $k$ of integers $n$ satisfying

(2) $\quad n \leqslant x, \quad n \equiv b(\bmod a), \quad n \equiv 0(\bmod p), \quad x^{1-1 / 2} l<p<x^{1-1 / 2 l+1}$ for an $l \geqslant l_{x}$ does not exceed $\sum_{x \geqslant p>x^{1-2}-l x}\left(\left[\frac{x}{p a}\right]+1\right)$, thus by theorems of

Mertens and Chebyshev

$$
k<\frac{c_{1} x}{a 2^{l_{x}}}+\frac{c_{2} x}{\log x}
$$

and by the definition of $l_{x}$

$$
k<\frac{c_{3} x}{\log x}
$$

Denote the numbers satisfying (2) for an $l \geqslant l_{x}$ by $a_{1}<a_{2}<\ldots$ $<a_{k} \leqslant x$. Since for all $y \leqslant x, v(y)<e_{4} \log x / \log \log x$ (from the prime number theorem or from more elementary results), we have

$$
\sum_{l \geqslant l_{x}} A_{l}^{\prime}(x) \leqslant \sum_{i=1}^{k} \nu\left(a_{i}\right)<\frac{c_{3} x}{\log x} \cdot \frac{c_{4} \log x}{\log \log x}<\frac{c_{5} x}{a}
$$

for $x>x_{1}(a)$.

For $l<l_{x}$ denote numbers satisfying (2) by $a_{1}^{(l)}<a_{2}^{(l)}<\ldots<a_{k_{l}}^{(l)}$. Similarly as for $k$ we have for $k_{l}$ the inequality

hence by $l<l_{x}$

$$
k_{l}<\frac{e_{6} x}{a 2^{l+1}}+\frac{c_{2} x}{\log x}
$$

$$
k_{l}<\frac{c_{7} x}{a \cdot 2^{l}} .
$$

We shall prove that for $l<l_{x}$ and sufficiently large $x$

$$
A_{l}^{\prime}(x)=\sum_{i=1}^{k_{l}} v_{l}\left(a_{i}^{(l)}+1\right)<\frac{c_{8} x}{a \cdot l^{2}}
$$

where $\nu_{l}(m)$ denotes the number of prime factors $>x^{1 / 2}{ }^{l+1}$ of $m$.

For this purpose, we split the summands of the sum (5) into two classes. In the first class are the integers $a_{i}^{(l)}$ for which $v_{l}\left(a_{i}^{(l)}+1\right) \leqslant 2^{l} / l^{2}$. From (4) it follows that the contribution of these integers $a_{i}^{(l)}$ to (5) is less than $c_{7} x / a l^{2}$. The integer in the second class satisfy $v_{l}\left(a_{i}^{(l)}+1\right)>2^{l} / l^{2}$. Thus these integers are divisible by more than $2^{l} / l^{2}$ primes $q>x^{1 / 2} l^{2+1}$. Thus the number of integers of the second class is less than

$$
\begin{aligned}
& \frac{x\left(\sum_{x^{1 / 2} 2^{l+1}<p_{\leqslant x}} \frac{1}{q}\right)^{\left[2^{l} / l^{2}\right]}}{\left[2^{l} / l^{2}\right] !}+\left[\frac{2^{l}}{l^{2}}\right] !<\frac{x\left(c_{9} l\right)^{\left[2^{l} l\right.}}{a\left[2^{l} / l^{2}\right] !}+\left[\frac{2 l^{l} x}{l_{x}^{2}}\right] ! \\
& <\frac{x}{a \cdot 5^{7}}+\left[\frac{2 \log x}{a \log \log x}\right] !<\frac{x}{a \cdot 4^{l}}
\end{aligned}
$$


for $l>c_{10}, x>x_{2}(a)$. By definition, $v_{l}\left(a_{i}^{(l)}+1\right)<2^{l+1}$. Thus, for $l>c_{10}$, the contribution of the numbers of the second class to (5) is $<x / a \cdot 2^{l-1}$; for $l \leqslant c_{10}$ the contribution is clearly $<2^{c_{10}+1} x$. Thus, for $l<l_{x}, x>x_{2}(a)$,

$$
A_{l}^{\prime}(x)<c_{8} x / a l^{2}
$$

and in view of (3) we have for $x>x_{0}(a)$

$$
\sum_{l=1}^{\infty} A_{l}^{\prime}(x)<\frac{c_{5} x}{a}+\sum_{l<l_{x}} \frac{c_{8} x}{a l^{2}}<\frac{c x}{a}
$$

which proves the Lemma.

Proof of the theorem. Let $\varepsilon$ be a positive number and let a sequence $a_{i}(i=1,2, \ldots, h)$ be given. that

By condition 2 we can find positive integers $N_{0}, N_{1}, \ldots, N_{h}$ such

(6) $\left(N_{i},(h+1) !\right)=1 \quad(i=0,1, \ldots, h), \quad\left(N_{i}, N_{j}\right)=1 \quad(0 \leqslant i<j \leqslant h)$,

$$
f\left(N_{0}\right)>c_{1}+\max _{1 \leqslant i \leqslant h}\left\{f(i+1)-\sum_{j=1}^{i} a_{j}\right\}
$$

and

$$
\left|f\left(N_{i}\right)-\left\{f\left(N_{0}\right)-f(i+1)+\sum_{j=1}^{i} a_{j}\right\}\right|<\frac{1}{4} \varepsilon \quad(1 \leqslant i \leqslant h) ;
$$

hence

$$
\left|f\left((i+1) N_{i}\right)-f\left(i N_{i-1}\right)-a_{i}\right|<\frac{1}{2} \varepsilon \quad(1 \leqslant i \leqslant h) .
$$

Let $k_{1}$ be the greatest prime factor of $N_{0} N_{1} \ldots N_{h}$. Put $\mu=$ $\varepsilon / \sqrt{96 h c}$ ( $c$ is the constant of the Lemma). By condition $1, \sum_{|f(p)| \geqslant \mu}(1 / p)$ is convergent. Since $\sum_{p}\left(1 / p^{2}\right)$ is also convergent, there exists a $k_{2}$ such that

$$
\sum_{\substack{\mid f(p)) \geqslant \gtrless \\ p>k_{2}}} \frac{1}{p}+\sum_{p>k_{2}} \frac{1}{p^{2}}<\frac{1}{3(h+1)} .
$$

Finally by condition 1 there exists a $k_{3}$ such that

$$
\sum_{\substack{|f(p)|<\mu \\ p>k_{3}}} \frac{f(p)^{2}}{p}<\frac{\varepsilon^{2}}{48 h}
$$

\section{Let us put}

$$
k=\max \left(k_{1}, k_{2}, k_{3}\right), \quad N=N_{1} N_{2} \ldots N_{h}, \quad P=\prod_{\substack{p<k \\ p \nmid N}} p, Q=(h+1) ! N^{2} P
$$

and let us consider the following system of congruences

$$
n \equiv 1(\bmod (h+1) ! P), \quad n \equiv-i+N_{i}\left(\bmod N_{i}^{2}\right), \quad 0 \leqslant i \leqslant h .
$$

By (6) and the Chinese Remainder Theorem there exists a number $n_{0}$ satisfying these congruences.

It is easy to see that

(10) for every integer $t$ the numbers $\left(Q t+n_{0}+i\right) /(i+1) N_{i}(i=1,2, \ldots, h)$ are integers which are not divisible by any prime $\leqslant k$;

(11) the number of terms not exceeding $x$ of the arithmetical progression $Q t+n_{0}$ is $x / Q+O(1)$.

In order to prove Theorem 1 we shall estimate the number of integers $n$ of the progression $Q t+n_{0}$ which satisfy the inequalities

(12) $n \leqslant x, \quad \sum_{i=1}^{h}\left(f(n+i)-f(n+i-1)-f\left((i+1) N_{i}\right)+f\left(i N_{i-1}\right)\right)^{2}>\frac{1}{4} \varepsilon^{2}$.

We divide the set of integers $n \equiv n_{0}(\bmod Q)$ for which the inequalities (12) hold into two classes. Integers $n$ such that $n(n+1) \ldots(n+h)$ is divisible by a prime $p>k$ with $|f(p)| \geqslant \mu$, or by $p^{2}, p>k$, are in the first class and all other integers are in the second class.

(13) The number of integers $n \leqslant x, n \equiv r(\bmod Q)$ which are divisible by a given integer $d>0$ is equal to $x / d Q+O(1)$ for $(d, Q)=1$,

hence the number of integers $n \leqslant x, n \equiv n_{0}(\bmod Q)$ of the first class is less than

$$
(h+1) \frac{x}{Q}\left(\sum_{\substack{p>k \\|f(p)| \geq \mu}} \frac{1}{p}+\sum_{p>k} \frac{1}{p^{2}}\right)+O\left(\sum_{p \leqslant x+h} 1+\sum_{p^{2} \leqslant x+h} 1\right) .
$$

By the inequality (8) and the definition of $k$ this number is less than $\frac{1}{3} x / Q+o(x)$.

For the integers of the second class, by remark (10) we have

$$
\begin{aligned}
\sum_{n}^{\prime \prime} \sum_{i=1}^{h}\left(f(n+i)-f(n+i-1)-f\left((i+1) N_{i}\right)+f\left(i N_{i-1}\right)\right)^{2} & =S=\sum_{n}^{\prime \prime} \sum_{i=1}^{h}\left\{\sum_{\substack{p \mid n+i \\
p>k}} f(p)-\sum_{\substack{p \mid++i-1 \\
p>k}} f(p)\right\}^{2},
\end{aligned}
$$


where $\Sigma^{\prime \prime}$ means that the summation runs through the integers of the second class. In view of remark $(13)$, since $(Q, p)=1$ we have

$$
\begin{aligned}
& S \leqslant \sum_{n=n_{0}(\bmod Q)} \sum_{i=1}^{n}\left\{\sum_{\substack{p \mid n+i \\
n \leqslant k}} f(p)-\sum_{\substack{p|n+i-1 \\
p>k,| f(p) \mid<\mu}} f(p)\right\}^{2} \\
& =\sum_{\substack{x+h>p>k \\
|f(p)|<\mu}} f^{2}(p)\left(\frac{2 h x}{Q p}+O(1)\right)+ \\
& +\sum_{\substack{n \equiv n_{0}(\bmod Q) \\
n \leqslant x}} \sum_{i=1}^{n}\left\{2 \sum_{\substack{p q|n+i, q>p>>k\\
| f(p)|<\mu,| f(q) \mid<\mu}} f(p) f(q)+\right. \\
& \left.+2 \sum_{\substack{p q|n+i-1, q>p>k\\
| f(p)|<\mu,| f(q) \mid<\mu}} f(p) f(q)-2 \sum_{\substack{p|n+i, q| n+i-1, q>k \\
p>k,|f(p)|<\mu,|f(q)|<\mu}} f(p) f(q)\right\} \\
& \leqslant \frac{2 h x}{Q} \sum_{p>k,|f(p)|<\mu} \frac{f^{2}(p)}{p}+\sum_{\substack{n=n_{0}(\bmod Q) \\
n \leqslant x}} \sum_{i=1}^{n} 2 \sum_{\begin{array}{c}
p|n+i, q| n+i-1 \\
p q>x, p>k, q>k \\
|f(p)|<\mu,|f(q)|<\mu
\end{array}}|f(p) f(q)|+ \\
& +O\left(\sum_{\substack{p \leq x+h \\
|f(p)|<\mu}} f^{2}(p)+\sum_{\substack{p>q>k, p q \leqslant x+h \\
|f(p)|<\mu,|f(q)|<\mu}}|f(p) f(q)|\right) .
\end{aligned}
$$

Thus finally from (9), Lemma, the equality $\mu^{2}=\varepsilon^{2} / 96 h c$ and from the fact that the number of integers of the form $p q$ not exceeding $x+h$ is $o(x)$, we get

$$
S<\frac{\varepsilon^{2}}{12} \cdot \frac{x}{Q}+o(x) .
$$

Thus the number of integers of the second class is less than $\frac{1}{3} x / Q+o(x)$. Hence there exist less than $\frac{2}{3} x / Q+o(x)$ positive integers $n \leqslant x$, $n \equiv n_{0}(\bmod Q)$ for which

$$
\sum_{i=1}^{h}\left(f(n+i)-f(n+i-1)-f\left((i+1) N_{i}\right)+f\left(i N_{i-1}\right)\right)^{2}>\frac{1}{4} \varepsilon^{2} .
$$

Therefore by (11) there exist more than $\frac{1}{3} x / Q+o(x)$ positive integers $n \leqslant x$, for which

$$
\sum_{i=1}^{h}\left(f(n+i)-f(n+i-1)-f\left((i+1) N_{i}\right)+f\left(i N_{i-1}\right)\right)^{2} \leqslant \frac{1}{4} \varepsilon^{2}
$$

and then

$$
\left|f(n+i)-f(n+i-1)-f\left((i+1) N_{i}\right)+f\left(i N_{i-1}\right)\right| \leqslant \frac{1}{2} \varepsilon \quad(i=1,2, \ldots, h) .
$$

In view of (7), the proof is complete.

THEOREM 2. Let $f(n)$ be an additive function satisfying the conditions of Theorem 1 and such that partial sums of $\Sigma(\|f(p)\| / p)$ are bounded:

$$
A>\left|S_{k}\right|, \quad S_{k}=\sum_{p \leqslant k} \frac{\|f(p)\|}{p} .
$$

Then for any given natural number $h$ there exists a number $c_{h}$ such that for any $\varepsilon>0$ and every sequence of $h$ numbers: $a_{1}, a_{2}, \ldots, a_{h} \geqslant c_{h}$, there exist more than $C(a, \varepsilon) x$ positive integers $n \leqslant x$, for which

$$
\left|f(n+i)-a_{i}\right|<\varepsilon \quad(i=1,2, \ldots, h) .
$$

$C(a, \varepsilon)$ is a positive constant, depending on $\varepsilon$ and $a_{i}$.

Proof. Let $\varepsilon$ be a positive number, $c_{h}=c_{1}+\dot{m} a x f(i)$ and let a sequence $a_{i} \geqslant c_{h}(i=1,2, \ldots, h)$ be given.

By condition 2 we can find positive integers $N_{1}, N_{2}, \ldots, N_{h}$ such

(16) $\quad\left(N_{i}, h !\right)=1 \quad(i=1,2, \ldots, h), \quad\left(N_{i}, N_{j}\right)=1 \quad(1 \leqslant i<j \leqslant h)$

$$
\left|f\left(N_{i}\right)-a_{i}+f(i)\right|<\frac{1}{2} \varepsilon \quad(i=1,2, \ldots, h) .
$$

Let $k_{1}$ be the greatest prime factor of $N_{1} N_{2} \ldots N_{h}$. Let $C$ be an absolute constant such that

$$
\sum_{y \leqslant p<\varepsilon} \frac{1}{p}<C \log \frac{\log z}{\log y} \quad \text { for all } \quad z>y>1 .
$$

Put $\mu=\varepsilon / 7 C \sqrt{h}$. By condition $1, \sum_{|f(p)| \geqslant \mu}(1 / p)$ is convergent. Since $\sum\left(1 / p^{2}\right)$ is also convergent, there exists a $k_{2}$ such that

$$
\sum_{|f(p)| \geqslant \mu, p>k_{2}} \frac{1}{p}+\sum_{p>k_{2}} \frac{1}{p^{2}}<\frac{1}{3 h}
$$

By condition 1 there exists also a $k_{3}$ such that

$$
\sum_{p>k_{3},|f(p)|<\mu} \frac{f(p)^{2}}{p}<\frac{\varepsilon^{2}}{24 h} .
$$

Put $\eta=\varepsilon / \sqrt{96 h}, B=A+1 / 3 h$ and denote by $I_{v}$ the interval

$$
\left[\nu \eta-\frac{1}{2} \eta, \nu \eta+\frac{1}{2} \eta\right], \quad v=0, \pm 1, \pm 2, \ldots, \pm[B / \eta+1]
$$


and let $k_{v}$ be the least integer $k>\max \left(k_{1}, k_{2}, k_{3}\right)$ such that $\sum_{n \leqslant k, f(f) \mid k k \mu}(f(p) / p)$ $\epsilon I_{v}$ if such integers $k$ exist, otherwise let $k_{v}=1$.

Now if $\sum_{p \leqslant x+h,|f(p)|<\mu}(f(p) / p) \in I_{v_{x}}$-by the condition (14) and by (18) such $v_{x}$ certainly exists-we put $k_{v_{x}}=k$ and then we get

$$
\left|\sum_{\substack{x+h \geq p>k \\|f(p)|<\mu}} \frac{f(p)}{p}\right|<\eta, \quad k \leqslant \max _{|\nu| \leqslant[B \mid \eta]+1} k_{v}=\bar{k} .
$$

Let $\Sigma^{\prime}$ denote that the summation runs through all primes $p, q$ satisfying conditions $p>q>k, p q \leqslant x+h,|f(p)|<\mu,|f(q)|<\mu$. From (20) we get

$$
\begin{aligned}
& \text { (21) } \quad 2 \sum^{\prime} \frac{f(p) f(q)}{p q} \leqslant\left(\sum_{\substack{x+h \geqslant p+k \\
|f(p)|<\mu}} \frac{f(p)}{p}\right)^{2}+\sum_{x+h \geqslant p>\sqrt{x+h}} \frac{\mu}{p} \sum_{\substack{x+h \geqslant q>\frac{x+h}{p} \\
\text {. }}} \frac{\mu}{q} \\
& \leqslant \frac{\varepsilon^{2}}{96 h}+\sum_{l=2}^{\infty} \sum_{(x+h+1)^{1-1 / 2} l>p \geqslant(x+h+1)^{1-1 / 2}} \frac{\mu}{p} \sum_{x+h \geqslant q>\frac{x+h}{p}} \frac{\mu}{q} \\
& \leqslant \frac{\varepsilon^{2}}{96 h}+\mu^{2} C^{2} \sum_{l=2}^{\infty} \frac{l}{2^{l}}=\frac{\varepsilon^{2}}{96 h}+\mu^{2} C^{2} \frac{3}{2}<\frac{\varepsilon^{2}}{24 h} \text {. }
\end{aligned}
$$

Let us put $N=N_{1} N_{2} \ldots N_{h}, \quad P=\prod_{p \leqslant h, p \nmid N} p$,

$$
Q=h ! N^{2} P \leqslant h ! N^{2} \prod_{p \leqslant \bar{k}, p+N} p=\bar{Q}
$$

and let us consider the following system of congruences:

$$
n \equiv 0(\bmod h ! P), \quad n \equiv-i+N_{i}\left(\bmod N_{i}^{2}\right) .
$$

By (16) and the Chinese Remainder Theorem there exists a number $n_{0}$ satisfying these congruences.

It is easy to see, that

(23) for every integer t the numbers $\frac{Q t+n_{0}+i}{i N_{i}}(i=1,2, \ldots, h)$ are integers, which are not divisible by any prime $\leqslant k$.

Analogously, as in the proof of Theorem 1, we shall estimate the number of integnss $n$ of the progression $Q t+n_{0}$, which satisfy the inequalities

$$
n \leqslant x, \quad \sum_{i=1}^{h}\left(f(n+i)-f\left(i N_{i}\right)\right)^{2}>\frac{1}{4} \varepsilon^{2} .
$$

We divide the set of integers $n \equiv n_{0}(\bmod Q)$, for which the inequalities (24) hold, into two classes. Integers $n$ such that $(n+1)(n+2) \ldots$ $(n+h)$ is divisible by a prime $p>k$ with $|f(p)| \geqslant \mu$ or by $p^{2}, p>\bar{k}$, are in the first class and all others integers are in the second class.

By remark (13) the number of integers $n \leqslant x, n \equiv n_{0}(\bmod Q)$ of the first class is less than

$$
h \frac{x}{Q}\left(\sum_{p>k, \mid\langle(p)| \geqslant \mu} \frac{1}{p}+\sum_{p>k} \frac{1}{p^{2}}\right)+O\left(\sum_{p \leqslant x+h} 1+\sum_{p^{2} \leqslant x+h} 1\right) .
$$

By the inequality (18) and the definition of $k$ this number is less than $\frac{1}{3} x / Q+o(x)$.

For the integers of the second class, by remark (23), we have

$$
\sum_{i=1}^{h}\left(f(n+i)-f\left(i N_{i}\right)\right)^{2}=\sum_{i=1}^{h}\left(\sum_{p \mid n+i, p>k} f(p)\right)^{2}
$$

and

$$
\sum_{n}^{\prime \prime} \sum_{i=1}^{h}\left(f(n+i)-f\left(i N_{i}\right)\right)^{2}=\sum_{n}^{\prime \prime} \sum_{i=1}^{h}\left(\sum_{p \mid n+i, p>k} f(p)\right),
$$

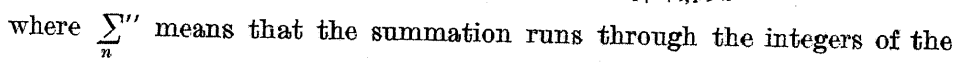
second class. In view of remark (13), we have

$$
\begin{aligned}
& \sum_{n}^{\prime \prime} \sum_{i=1}^{h}\left(f(n+i)-f\left(i N_{i}\right)\right)^{2} \leqslant \sum_{\substack{n \equiv n_{0}(\bmod Q) \\
n \leqslant x}} \sum_{i=1}^{h}\left(\sum_{p \mid n+i, p>k} f(p)\right)^{2} . \\
& =\sum_{\substack{x+h>p>k \\
|f(p)|<\mu}} f^{2}(p)\left(\frac{h x}{Q p}+O(1)\right)+2 \sum^{\prime} f(p) f(q)\left(\frac{h x}{Q p q}+O(1)\right) \\
& \leqslant \frac{h x}{Q}\left(\sum_{p>k,\langle f(p)|<\mu} \frac{f^{2}(p)}{p}+2 \sum^{\prime} \frac{f(p) f(q)}{p q}\right)+ \\
& +O\left(\sum_{\substack{p \leq x+h \\
|f(p)|<\mu}} f^{2}(p)+\sum^{\prime}|f(p) f(q)|\right) .
\end{aligned}
$$

Thus, finally from (19), (21) and from the fact that the number of integrers of the form $p q$ not exeeeding $x+h$ is $o(x)$ we gut

$$
\sum^{\prime \prime} \sum_{i=1}^{h}\left(f(n+i)-f\left(i N_{i}\right)\right)^{2}<\frac{\varepsilon^{2}}{12} \cdot \frac{x}{Q}+o(x) .
$$


Thus the number of integers of the second class is less than $\frac{1}{3} x / Q+o(x)$.

Hence, there exist less than $\frac{2}{3} x / Q+o(x)$ positive integers $n \leqslant x$, $n \equiv n_{0}(\bmod Q)$ for which

$$
\sum_{i=1}^{h}\left(f(b n+i)-f\left(i N_{i}\right)\right)^{2}>\frac{1}{4} \varepsilon^{2}
$$

By (11) and (22) there exist, therefore, more than $\frac{1}{3} x / \bar{Q}+o(x)$ positive integers $n \leqslant x$, for which

$$
\sum_{i=1}^{h}\left(f(n+i)-f\left(i N_{i}\right)\right)^{2} \leqslant \frac{1}{4} \varepsilon^{2}
$$

and then

$$
\left|f(n+i)-f\left(i N_{i}\right)\right| \leqslant \frac{1}{2} \varepsilon \quad(i=1,2, \ldots, h) .
$$

In view of (16) and (17), this completes the proof.

Theorem 2 is best possible. Assume only that there exists an $a$ and a $e>0$ so that the number of integers $n \leqslant x$ satisfyng $|f(n)|<a$ is greater than $c x$.

Then $\sum \frac{\|f(p)\|^{2}}{p}$ converges and $\sum \frac{\|f(p)\|}{p}$ has bounded partial sums.

In the paper [2], P. Erdös proved $\left({ }^{1}\right)$ the following theorem:

If there exist two constants $c_{1}$ and $c_{2}$ and an infinite sequence $x_{k} \rightarrow \infty$ so that for every $x_{k}$ there are at least $c_{1} x_{k}$ integers:

$$
1 \leqslant a_{1}<a_{2}<\ldots<a_{l} \leqslant x_{k}, \quad l \geqslant c_{1} x_{k},
$$

for which

$$
\left|f\left(a_{i}\right)-f\left(a_{j}\right)\right|<c_{2}, \quad 1 \leqslant i<j \leqslant l,
$$

then

$$
f(n)=\operatorname{alog} n+g(n), \quad \text { where } \quad \sum \frac{\|g(p)\|^{2}}{p}<\infty .
$$

In our case the conditions of this theorem are clearly satisfied and, in fact, we elearly must have $a=0$. This implies that

$$
\sum \frac{\|f(p)\|^{2}}{p}<\infty
$$

( $\left.{ }^{1}\right)$ The proof of Lemma 8 [2] is not clear and on p. 15 needs more details similar to these given above.
Assume now that $\sum(\|f(p)\| / p)$ does not have bounded partial sums. Let e.g. $\sum_{p<x}(\|f(p)\| / p)=A, A$ large. Then by the method of Turán ([5], cf. also [2]) we obtain

$$
\sum_{n=1}^{x}(f(n)-A)^{2}<c_{3} x
$$

which implies that $|f(n)-A|<A-a$ for all but $\eta x$ integers $n \leqslant x$, where $\eta=c_{3} /(A-a)^{2}$. For suffieiently large $A$, it contradiets the assumption that $|f(n)|<a$ has $c x$ solutions $n \leqslant x$, thus the proof is complete.

In Theorem 1 one can replace $\sum\left(\|f(p)\|^{2} / p\right)<\infty$ by: there is an $\alpha$ so that if we put $f(n)-a \log n=g(n)$ then $\sum\left(\|g(p)\|^{2} / p\right)<\infty$. We think that here we again have a necessary and sufficient condition, but we cannot prove this. In fact, we conjecture that if there exist an $a$ and an $c>0$ such that the number of integers $n \leqslant x$ satisfying $|f(n+1)-f(n)|<a$ is $>c x$, then

$$
f(n)=\alpha \log n+g(n) \quad \text { with } \quad \sum \frac{\|g(p)\|^{2}}{p}<\infty .
$$

\$ 2. The proof of Theorem 2 is very similar to the proof of Lemma 1 of P. Erdös' paper [1]. Using ideas and results from that paper we can prove the following theorem.

THEOREM 3. Let $f(n)$ be an additive function satisfying condition 1 of Theorem 1 and let $\sum_{f(p) \neq 0}(1 / p)$ be divergent, $\sum(\|f(p)\| / p)$ convergent, then the distribution function of $h$-tuples $\{f(m+1), f(m+2), \ldots, f(m+h)\}$ exists, and it is a continuous function.

Proof. We denote by $N\left(f ; c_{1}, c_{2}, \ldots, c_{h}\right)$ the number of positive integers $m$ not exceeding $n$, for which

$$
f(m+i) \geqslant c_{i}, \quad i=1,2, \ldots, h,
$$

where $c_{i}$ are given constants.

It is sufficient to consider, as in [1], the special case in which, for any $\alpha, f\left(p^{a}\right)=f(p)$, so that

$$
f(m)=\sum_{p \mid m} f(p) .
$$

Let us also consider the function $f_{k}(m)=\sum_{p \mid m, n \leqslant k} f(p)$. We are going to show that the sequence $N\left(f_{k} ; c_{1}, c_{2}, \ldots, c_{h}\right) / n$ is convergent. For, if we denote by $A_{i, j}\left(j \leqslant j_{0, i}\right)$ the squarefree integers whose prime factors are not greater than $k$, and for which $f_{k}\left(A_{i, j}\right) \geqslant c_{i}$, we can see that the integers $m$ for which

$$
f_{k}(m+i) \geqslant c_{i} \quad(i=1,2, \ldots, h)
$$


are distributed periodically with the period $\prod_{\substack{1 \leqslant i \leqslant h \\ 1 \leqslant j \leqslant j_{0}, i}} A_{i, j}$. Hence $N\left(f_{k} ; c_{1}\right.$ $\left.e_{2}, \ldots, c_{h}\right) / n$ has a limit.

To prove the existence of a limit of $N\left(f ; c_{1}, c_{2}, \ldots, c_{h}\right) / n$ it is sufficient to show that for arbitrary $\varepsilon>0$ there exists $k_{0}$ such that for every $k>k_{0}$ and $n>n(\varepsilon)$

$$
\left|N\left(f ; c_{1}, c_{2}, \ldots, c_{h}\right)-N\left(f_{k} ; c_{1}, c_{2}, \ldots, c_{h}\right)\right| / n<\varepsilon .
$$

To show this, it is enough to prove that the number of integers $m \leqslant n$ for which there exists $i \leqslant h$ such that $f_{k}(m+i)<c_{i}$ and $f(m+i) \geqslant c_{i}$ or $f_{k}(m+i) \geqslant c_{i}$ and $f(m+i)<c_{i}$ is less than $\varepsilon h n$. But it is an immediate consequence of the analogous theorem for $h=1$ proved in [1], p. 123 .

In order to prove that the distribution function is continuous we must show that for every $\varepsilon>0$, there exists a $\delta>0$, such that

$$
\Delta=N\left(f ; c_{1}-\delta, c_{2}-\delta, \ldots, c_{h}-\delta\right)-N\left(f ; c_{1}+\delta, c_{2}+\delta, \ldots, c_{h}+\delta\right)<\varepsilon .
$$

Now

$$
\begin{aligned}
\Delta=\sum_{i=1}^{h}\left\{N\left(f ; c_{1}+\delta, \ldots, c_{i-1}+\delta, c_{i}-\delta, \ldots, c_{h}-\delta\right)-\right. \\
\left.\quad-N\left(f ; c_{1}+\delta, \ldots, c_{i}+\delta, c_{i+1}-\delta, \ldots, c_{h}-\delta\right)\right\}
\end{aligned}
$$

and by Lemma 2 of [1] each term of this sum is less than $\varepsilon / h$ for suitably chosen $\delta$. This completes the proof.

We conclude from Theorems 2 and 3 that if an additive function $f$ satisfies conditions $1,2, \sum_{f(p) \neq 0}(1 / p)$ is divergent and $\sum(\|f(p)\| / p)$ convergent, then the distribution function of $\{f(m+1), \ldots, f(m+h)\}$ exists, is continuous and strictly decreasing on some half straight-line, thus the sequence of integers $n$ for which inequality (15) holds has a positive density. Similarly we can prove the following:

THEOREM 4. Assume that $\sum_{f(p) \neq 0} \frac{1}{p}=\infty$ and that $\sum \frac{\|f(p)\|^{2}}{p}<\infty$. then $\{f(n+1)-f(n), f(n+2)-f(n+1), \ldots, f(n+k)-f(n+k-1)\} \quad$ has a continuous distribution function.

It is easy to see that condition 2 can be replaced by the conditions

$$
\lim _{p \rightarrow \infty} f(p)=0 \quad \text { and } \quad \sum_{p}^{Y}|f(p)|=\infty .
$$

\$3. $\mathrm{X}$. Wang proved in [6] that the number $N$ of primes $p<x$ satisfying

$$
\left|\frac{\varphi(p+\nu+1)}{\varphi(p+\nu)}-a_{\nu}\right|<\varepsilon, \quad 1 \leqslant \nu \leqslant k
$$

is greater than

$$
c(a, \varepsilon) \frac{x}{(\log x)^{k+2} \log \log x} .
$$

By our methods we can obtain in that case

$$
N>c_{1}(a, \varepsilon) \frac{x}{\log x}
$$

After having passed to the additive function $\log (\varphi(n) / n)$ the proof is similar to the proof of Theorem 1 . We use the fact, that $\log (\varphi(n) / n)$ is always negative, and apply the asymptotic formula for the number of primes in arithmetical progression instead of (11) and the Brun-Titchmarsh theorem instead of (13).

We can also prove that there exists distribution function $N\left(c_{1}, c_{2}, \ldots, c_{k}\right)$ defined as

$$
\lim _{x \rightarrow \infty} \frac{1}{\pi(x)} N\left(p<x ; \frac{\varphi(p+v)}{p+v} \geqslant c_{v}, \quad v=1,2, \ldots, k\right) .
$$

\section{References}

[1] P. Erdös, On the density of some sequences of numbers, III, J. London Math. Soc. 13 (1938), pp. 119-127.

[2] P. Erdös, On the distribution function of additive functions, Ann. of Math. 47 (1946), pp. $4-20$.

[3] A. Schinzel and Y. Wang, A Note on some properties of the functions $\varphi(n), \sigma(n)$ and $\theta(n)$, Bull. Acad. Polon. Sci. Cl. III 4 (1956), 207-209 and Ann. Pol. Math. 4 (1958), pp. $201-213$.

[4] Shao Pin Tsung, On the distribution of the values of a class of arithmetical functions, Bull. Acad. Polon. Sei., Cl. III 4 (1956), pp. 569-572.

[5] P. Turán, On a theorem of Hardy and Ramanujan, J. London Math. Soc. 9 (1934), pp. 274-276.

[6] Y. Wang, A note on some properties of the arithmetical functions $\varphi(n), \sigma(n)$ and $d(n)$, Acta Math. Sinica, 8 (1958), pp. 1-11.

Reçu par la Rédaction le 1.10.1960 